\title{
LAUTVERSCHIEBUNGEN IM ISLÄNDISCHEN
}

Lautverschiebungen sind Lautveränderungen, die ganze Lautreihen verschiedener Artikulationsstellen erfassen. Das Ergebnis einer Lautverschiebung ist die Phonologisierung von Allophonen bzw. die Umphonologisierung von Einheiten des Lautsystems. Lautverschiebungen haben im Verlauf der Geschichte in vielen Sprachgemeinschaften stattgefunden, u.a. auch in den germanischen Sprachen. Sehr bekannt und gut untersucht ist die Erste Germanische Lautverschiebung vom Indogermanischen zum Germanischen und die Zweite Deutsche Lautverschiebung.

Isländisch gilt als die stabilste und konservativste germanische Sprache, da es sowohl die Kasusformen des Nomens und des Adjektivs als auch die Konjugationsformen des Verbs besser als andere germanische Sprachen bewahrt hat. Das hat eine Art Mythos geschaffen. Man spricht sogar von diachronischer Stabilität (Groenke 1983). Meiner Ansicht nach ist es jedoch übertrieben, von diachronischer Stabilität zu sprechen. Viele Substantive kommen in erstaunlicher Formenvielfalt vor. Für einzelne Kasus gibt es mehrere Formen und es gibt sogar Fälle, in denen ein Substantiv zu mehr als einem Genus gehört, wie z.B. skúr, Regenschauer', das feminin oder maskulin sein kann.

In der phonetischen Entwicklung ist das Isländische alles andere als konservativ. Es haben sehr tiefgreifende Änderungen stattgefunden, die die Sprache derart umgestaltet haben, dass eine Verständigung mit Sprechern des Altisländischen wahrscheinlich nicht ohne weiteres möglich wäre, gesetzt der hypothetische Fall, dass wir solche Sprecher hören könnten.

Im Folgenden möchte ich versuchen, einen Überblick über die wichtigsten Änderungen vom Altisländischen zum modernen Isländischen zu geben. Ich hoffe dadurch, die Meinung zu relativieren, dass Isländisch so stabil ist und einen realitätsnahen Eindruck von der Sprache vermitteln zu können.

\section{Verlust der segmentalen Quantität}

Der Verlust der segmentalen Quantität wird im allgemeinen im 16. Jahrhundert angesetzt, obwohl er früher geschehen sein könnte. Diese Änderung wurde sehr folgenreich. Sie bedeutete die Phonologisierung der Vokalqualitäten, die bisher nur allophonisch gewesen waren. Die Quantität betraf aber weiterhin Vokale und Konsonanten, wurde aber eine Eigenschaft der Silbe (Pórólfsson 1929). 
Im Altisländischen gab es vier Silbentypen aus quantitativer Sicht:

(1.) /V:C:/ langer Vokal und langer Konsonant átta [a:t:a] "acht"

(2.) $\quad / \mathrm{V}: \mathrm{C} / \quad$ langer Vokal und kurzer Konsonant áta [a:t(h)a] "Nahrung (für Fische)"

(3.) /VC:/ kurzer Vokal und langer Konsonant bzw. Konsonantengruppe /VCC/ atti [at:i] "(er)spornte an", Prät. von etja "anspornen" vildi [vildi] "(er)wollte", Prät. von vilja "wollen"

(4.) / VC/ kurzer Vokal und kurzer Konsonant ana [ana] "stürmen, schnell laufen"

Die Silbentypen (1) und (4) wurden eliminiert. Es blieben nur die Silbentypen (2) und (3). Kurze Vokale konnten von nun an nur vor langen Konsonanten oder vor einer Konsonantengruppe vorkommen, während lange Vokale nur vor kurzen Konsonanten stehen konnten oder alleine in offener Silbe. Außerdem wurde die Quantität auf die betonte (hauptbetonte oder nebenbetonte) Silbe eingeschränkt. Unbetonte Silben waren quantitativ neutral.

Es gab aber zu diesen Regeln eine Ausnahme. Wenn das erste Graphem einer postvokalischen Konsonantengruppe $\langle\mathrm{p} \mathrm{t} \mathrm{k} \mathrm{s}>$ und zweite Graphem $\langle\mathrm{v}$ j $\mathrm{r}\rangle$ war, blieb der Vokal lang. Beispiele sind in Tabelle 1 zu sehen.

\begin{tabular}{|lll|}
\hline lepja & {$[$ le:pja] } & "schlürfen" \\
kipra & {$\left[\mathrm{c}^{\mathrm{h}}\right.$ I:pra] } & "zucken" \\
(upp)götva & {$[($ Yhp)kœ:tva] } & "entdecken" \\
etja & [e:tja] & "anspornen" \\
sætra & [sai:tra] & "süß (Gen. Pl.)" \\
skrökva & [skrœ:kva] & "lügen" \\
veikra & [vei:kra] & "krank (Gen. Pl.)" \\
vekja & [ve:ca] & "wecken" \\
hösvir & [hœ:svir] & "Sklave" \\
Esja & [e:sja] & (Ortsname) \\
lausra & {$[$ lœei:sra] } & "lose (Gen. Pl.)" \\
\hline
\end{tabular}

Tabelle 1: $\quad$ Lange Vokale vor einer Graphemgruppe.

Die Transkription ist die des modernen Isländisch

Allerdings sind diese Ausnahmen nicht so bedeutend wie sie häufig dargestellt werden. Die Graphemgruppe $\langle\mathrm{kj}\rangle$ ist phonetisch gesehen nur ein palataler Konsonant [c], vor dem der Vokal lang sein muß und die Graphemgruppe <sv> kommt faktisch nur in einem einzigen veralteten Wort hösvir ,Sklave' vor. 
In der gesprochenen Sprache können heutzutage kurze Vokale vor allen diesen Graphemgruppen (außer $\langle\mathrm{kj}>$ ) stehen, so dass man sagen kann, dass die Lautveränderung zum Ende gekommen ist. Das Vorhandensein des kurzen Vokals ist unterschiedlich häufig, aber es ist möglich. In häufig verwendeten Wörtern ist der kurze Vokal häufiger als in seltenen Wörtern.

Vor der Aufgabe der segmentalen Quantität wird Altisländisch einen Rhythmus gehabt haben, der an das heutige Finnisch oder Estnisch erinnert. Daran kann gemessen werden, wie bedeutsam der Verlust der segmentalen Quantität gewesen sein muß.

\section{Das Vokalsystem}

Im Vokalsystem gab es eine bedeutende Änderung, die daraus resultierte, dass die Vokalqualität distinktiv wurde und nicht mehr die Vokalquantität. Das altisländische Vokalsystem hatte folgende Form:

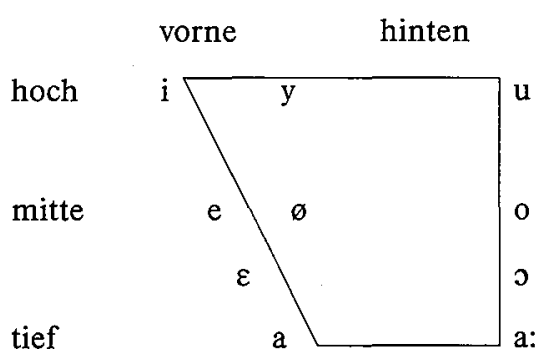

Diphthonge: au ei ey [oy]

Alle Vokale konnten lang und kurz unabhängig von der Silbenstruktur vorkommen. Bei den kurzen Vokalen fanden folgende Änderungen statt:

1. [i] wurde geöffnet $\mathrm{zu}[\mathrm{I}]$

2. [y] wurde entrundet und fiel mit dem [I] zusammen

3. [u] wurde nach vorne verlagert, geöffnet und bildete einen neuen Vokal [Y]

4. [ø] wurde $\mathrm{zu}[œ]$ geöffnet

5. [0] wurde nach vorne verlagert und fiel mit [œ] zusammen

6. [o] wurde geöffnet

Von den kurzen Vokalen blieb nur das [a] unverändert. Bei đen langen Vokalen fanden folgende Änderungen statt:

1. [y:] wurde entrundet und fiel mit [i] zusammen

2. [e:] wurde zu [je] diphthongiert

3. [ع:] wurde zu [ai] diphthongiert

4. [ø:] wurde geöffnet und fiel mit [ai] zusammen

5. [0:] und [a:] fielen zusammen und wurden zu [au] diphthongiert

6. [o:] wurde $\mathrm{zu}[\mathrm{ou}]$ diphthongiert 
Bei den langen Vokalen blieben nur [i:] und [u:] unverändert erhalten. Das Ergebnis dieser Veränderungen ist das moderne isländische Vokalsystem:

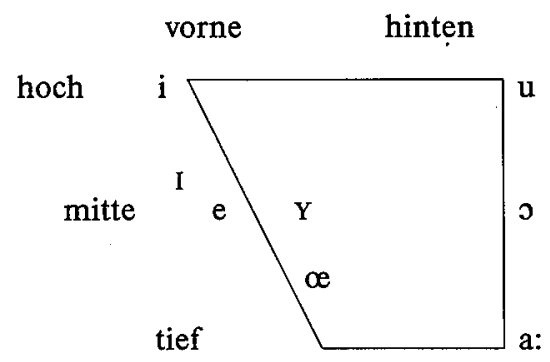

Außerdem gibt es die fünf Diphthonge: [ei ai öi au ou]. Jeder Vokal und jeder Diphthong kann kurz und lang in Abhängigkeit von der Silbenstruktur vorkommen. Die Diphthonge werden in dieser Hinsicht wie Monophthonge behandelt. Die neuen Klangfarben bedeuten ein völlig anderes Klangbild als im Altisländischen. Kombiniert mit der neuen rhythmischen Struktur handelt es sich um sehr bedeutende Veränderungen.

Das System ist aber asymmetrisch, da nur drei hintere Vokale fünf vorderen Vokalen gegenüber stehen. Die Tendenz der heutigen Aussprache, den Diphthong [ou] zu monophthongieren und als [o] zu sprechen, schafft eine teilweise Symmetrie.

Eine andere Tendenz war das flámceli "schiefes Sprechen", in dem die Vokalqualitäten [x] und [Y] aufgegeben wurden und zu [e] und [œ] wurden: pekja [Өe:ca]

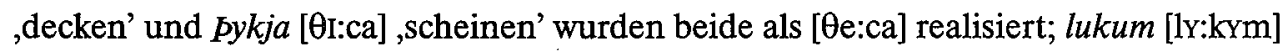
,(wir) beendeten' und lökum [lœ:kxm] ,Bettlaken' wurden beide als [lœ:krm] realisiert. Das Vokalsystem erlangte hierdurch eine Symmetrie, aber viele lexikalische Unterscheidungen gingen verloren. Deshalb wurde diese Aussprache verpönt und mit allen Mitteln von den Behörden und der Schule bekämpft. Sie ist heute weitgehend verschwunden, aber könnte noch in dem in Kanada gesprochenen Isländisch vorhanden sein (Bessason 1967 behandelt aber wenig die Aussprache).

\section{Das Konsonantensystem}

Die Veränderungen im Konsonantensystem sind sehr bedeutsam und führen letztendlich zu dem typologisch seltenen Konsonantensystem des modernen Isländischen, das in Tabelle $2 \mathrm{zu}$ sehen ist:

Tabelle 2. Das Konsonantensystem des modernen Isländischen

\begin{tabular}{|l|l|l|l|l|l|l|}
\hline & & Labial & dental & palatal & velar & Glottal \\
\hline Verschluß & Unaspiriert & $\mathrm{p}$ & $\mathrm{t}$ & $\mathrm{c}$ & $\mathrm{k}$ & \\
\hline & Aspiriert & $\mathrm{p}^{\mathrm{h}}$ & $\mathrm{t}^{\mathrm{h}}$ & $\mathrm{c}^{\mathrm{h}}$ & $\mathbf{k}^{\mathrm{h}}$ & \\
\hline
\end{tabular}




\begin{tabular}{|l|l|l|l|l|l|l|}
\hline Nasal & Stimmlos & $\mathrm{m}$ & $\mathrm{n}$ & $\mathrm{n}$ & $\mathbf{y}$ & \\
\hline & Stimmhaft & $\mathrm{m}$ & $\mathrm{n}$ & $\mathrm{n}$ & $\mathbf{y}$ & \\
\hline Liquid & Stimmlos & & $\mathrm{l} \mathrm{r}$ & & & \\
\hline & Stimmhaft & & $\mathbf{1} \mathrm{r}$ & & & \\
\hline Frikativ & Stimmlos & $\mathrm{f}$ & $\theta \mathrm{s}$ & $\mathrm{c}$ & $\mathrm{x}$ & $\mathbf{h}$ \\
\hline & Stimmhaft & $\mathrm{v}$ & $\mathrm{d}$ & $\mathbf{j}$ & $\mathrm{y}$ & \\
\hline
\end{tabular}

In diesem System, das nach dem Prinzip der relativen Artikulationsenergie verstanden als die Berührungsfläche zwischen den Organen geordnet ist, gibt es keine stimmhaften Verschlusslaute. Die beiden Verschlusslautreihen unterscheiden sich nur durch das Vorhandensein der Aspiration. Bei den Nasalen, Liquiden und Frikativen besteht aber eine Opposition zwischen stimmhaft und stimmlos. Die palatalen und velaren Nasale kommen nur vor homorganen Konsonanten vor und sind daher als Allophone der dentalen Nasale anzusehen. Die velaren Frikative gehören zu einem Phonem [x]. Das [s] hat keine stimmhafte Entsprechung.

Die Verschlusslaute bilden nur eine Opposition im Silbenanlaut wie in Tabelle 3 zu sehen ist.

Tabelle 3. Distinktive Oppositionen von Verschlußkonsonanten im Silbenanlaut

\begin{tabular}{|c|c|}
\hline$\langle\mathrm{b}\rangle=[\mathrm{p}]$ & $\langle\mathrm{p}\rangle=\left[\mathrm{p}^{\mathrm{h}}\right]$ \\
\hline bara [pa:ra] ,nur' & para [p $\left.{ }^{h_{2}}: r a\right]$, paaren' \\
\hline$\langle\mathrm{d}\rangle=[\mathrm{t}]$ & $\langle\mathrm{t}\rangle=\left[\mathrm{t}^{\mathrm{h}}\right]$ \\
\hline dæma [taima] ,beurteilen' & tæma [t ${ }^{\mathrm{h}}$ ai:ma] , leeren' \\
\hline$\langle\mathrm{gj}\rangle=[\mathrm{c}]$ & $\langle\mathrm{kj}\rangle=\left[\mathrm{c}^{\mathrm{h}}\right]$ \\
\hline gjósa [cou:sa] ,ausbrechen' & kjósa [c ${ }^{\mathrm{h}}$ ou:sa] ,wählen' \\
\hline$\langle\mathrm{g}\rangle=[\mathrm{k}]$ & $\langle\mathrm{k}\rangle=\left[\mathrm{k}^{\mathrm{h}}\right]$ \\
\hline gula [ky:la] ,gelb' (Akk.) & kula [k $\left.\mathrm{k}^{\mathrm{h}} \mathrm{Y}: \mathrm{la}\right]$,kühl werden' \\
\hline
\end{tabular}

Das moderne isländische Konsonantensystem ist das Ergebnis einer großen Lautverschiebung, deren erster Schritt der Verlust der Stimmhaftigkeit der alten $\langle\mathrm{b} d \mathrm{~g}\rangle$ war. Diese fielen mit den alten $\langle\mathrm{p} \mathrm{t} \mathrm{k}\rangle$ in allen Stellungen zusammen außer im Anlaut einer betonten Silbe (Tabelle 3). Es ist wahrscheinlich, dass die alten $\langle p t k\rangle$ aspiriert waren, denn in einem nördlichen Gebiet werden sie immer noch intervokalisch aspiriert gesprochen: tapa [ $\mathrm{t}^{\mathrm{h}} \mathrm{a}: \mathrm{p}^{\mathrm{h}} \mathrm{a}$ ] ,verlieren', láta [lau: $\mathrm{t}^{\mathrm{h}} \mathrm{a}$, lassen', moka [mo: $\mathrm{k}^{\mathrm{h}} \mathrm{a}$ ] ,schaufeln'. Das Beibehalten der Aspiration in der intervokalischen Stellung kann darauf hindeuten, dass die Silbengrenze vor dem Konsonanten liegt, obwohl die orthographischen Regeln der Silbentrennung genau das Gegenteil andeuten (Berg 2001). 
Die Tatsache, dass saga [sa:ya] ,Geschichte' heute mit stimmhaftem velaren Frikativ gesprochen wird, deutet unzweifelhaft darauf hin, dass das alte $\langle\mathrm{g}\rangle$ wirklich stimmhaft war. Das Gleiche würde für $\langle\mathrm{b} d\rangle$ gelten, aber wegen phonotaktischen Einschränkungen liegen uns keine Beispiele hier vor.

Durch den Verlust der Stimmhaftigkeit wären nun die langen $\langle\mathrm{bb}$ dd gg $\rangle$ identisch mit den alten langen <pp tt kk> geworden (Tabelle 4).

Tabelle 4. Rekonstruierte Aussprache der langen Verschlußlaute im Altisländischen

\begin{tabular}{|l|l|}
\hline$\langle\mathrm{pp}\rangle=[\mathrm{p}:]$ & $\langle\mathrm{bb}\rangle=[\mathrm{b}:]$ \\
\hline lappa [lap:a] ,verbessern' & labba [lab:a] ,gehen' \\
\hline$\langle\mathrm{tt}\rangle=[\mathrm{t}:]$ & $\langle\mathrm{dd}\rangle=[\mathrm{d}:]$ \\
\hline veittur [veit:ur] ,erteilt' (Part.M.) & veiddur [veid:ur] ,gefangen' (Part.M.) \\
\hline$\langle\mathrm{kk}\rangle=$ [k:] & $\langle\mathrm{gg}\rangle=$ [g:] \\
\hline sekkur [sek:ur] ,Sack' & seggur [seg:ur] ,Mann' \\
\hline
\end{tabular}

Dies ist aber nicht geschehen, weil vor den alten <pp tt $\mathrm{kk}>$ sich ein Hauch entwickelte, der häufig Präaspiration ${ }^{1}$ genannt wird. Daraus wurde eine Konsonantengruppe mit [h] als erstem Konsonanten und kurzem Verschlusskonsonanten an zweiter Stelle wie aus Tabelle $5 \mathrm{zu}$ ersehen ist:

Tabelle 5. Aussprache der ehemals langen Verschlußkonsonanten im modernen Isländischen

\begin{tabular}{|l|l|}
\hline$\langle\mathrm{pp}\rangle=[\mathrm{hp}]$ & $\langle\mathrm{bb}\rangle=[\mathrm{p}:]$ \\
\hline lappa [lahpa], verbessern' & labba [lap:a], gehen' \\
\hline$\langle\mathrm{tt}\rangle=[\mathrm{ht}]$ & $\langle\mathrm{dd}\rangle=[\mathrm{t}:]$ \\
\hline veittur [veihtyr] ,erteilt' (Part.M.) & veiddur [veit:Yr], gefangen' (Part.M.) \\
\hline$\langle\mathrm{kk}\rangle=[\mathrm{hk}]$ & $\langle\mathrm{gg}\rangle=[\mathrm{k}:]$ \\
\hline sekkur [sehkyr] ,Sack' & seggur [sek:Yr] ,Mann' \\
\hline
\end{tabular}

Dieser Hauch wurde auch auf die ursprünglichen Gruppen 〈tl tn pl pn kl kn> übertragen, die sonst gleich den Gruppen $<\mathrm{rl} 11 \mathrm{rn} n \mathrm{fl} \mathrm{fn}$ gn gl $>^{2}$ geworden wären, als deren erster Konsonant zu einem Verschlusslaut wurde. Tabelle 6 faßt diese Entwicklung zusammen.

1 Phonetisch gesehen ist dieser Hauch genau das gleiche Phänomen, wie das Ersetzen des [s] durch [h] im Spanischen: esto [ehto] ,dies', hasta [ahta] ,bis' usw. Es wäre auch möglich hier von Präaspiration zu sprechen, obwohl dies wegen der Beschreibungstradition der romanischen Sprachwissenschaft nicht gemacht wird.

2 Hier sollte auch erwähnt werden, dass in einem kleinen nördlichen Gebiet der erste Konsonant in den Gruppen <fð gð> zu Verschlusslaut wurde: hafði [hapdr], hatte', sagði [sakð̃I] ,sagte'. Im übrigen Sprachgebiet ist ein Frikativ vorhanden: [havðr] und [sayðr]. 
Tabelle 6.

Entwicklung einiger neuen und alten Konsonantengruppen im modernen Isländischen

\begin{tabular}{|c|c|}
\hline$\langle\mathrm{rl}\rangle\langle\mathrm{ll}\rangle=[\mathrm{tl}]$ & $\langle\mathrm{tl}\rangle=[\mathrm{htl}]$ \\
\hline hella [hetla], gießen' & vætla [vaihtla] ,sickern' \\
\hline \multicolumn{2}{|l|}{ varla [vatla] ,kaum' } \\
\hline$\langle\mathrm{rn}\rangle\langle\mathrm{nn}\rangle=[\mathrm{tn}]$ & $\langle\mathrm{tn}\rangle=[\mathrm{htn}]$ \\
\hline vænna [vaitna] ,beliebt' (Gen.Pl.) & vatna [vahtna] ,bewässern' \\
\hline \multicolumn{2}{|l|}{ varna [vatna], wehren' } \\
\hline$\langle\mathrm{fl}\rangle=[\mathrm{pl}]$ & $\langle\mathrm{pl}\rangle=[\mathrm{hpl}]$ \\
\hline afla [apla], erwerben' & epli [ehpli] ,Apfel' \\
\hline$\langle\mathrm{fn}\rangle=[\mathrm{pn}]$ & $\langle\mathrm{pn}\rangle=[\mathrm{hpn}]$ \\
\hline ofna [opna] ,Öfen' (Gen.Pl.) & opna [ohpna] ,öffnen' \\
\hline$\langle\mathrm{gn}\rangle=[\mathrm{kn}]$ & $\langle\mathrm{kn}\rangle=[\mathrm{hkn}]$ \\
\hline sagnir [saknir], Verben' & sakna [sahkna], vermissen' \\
\hline$\langle\mathrm{gl} \mid\rangle=[\mathrm{kl}]$ & $\langle\mathrm{kl}\rangle=[\mathrm{hkl}]$ \\
\hline seigla [seikla] ,Hartnäckigkeit' & Hekla [hehkla] Eigenname \\
\hline
\end{tabular}

Als letzter Schritt oder letzte Stufe dieser Lautverschiebung entstanden stimmlose Nasale und Liquide. Man kann annehmen, dass die Liquiden zuerst erfasst wurden, denn $\langle 1\rangle$ ist immer stimmlos vor $\langle t\rangle$ : valta ,wälzen' kann nur [valta] sein; *[valt $\left.{ }^{\mathrm{h}} \mathrm{a}\right]$ ist ausgeschlossen; $\langle\mathrm{r}\rangle$ ist ebenfalls immer stimmlos vor $\langle\mathrm{p} \mathrm{t} \mathrm{k} \mathrm{s}\rangle:$ harpa [harpa] ,Harfe', virtur [vırtyr] ,geschätzt', marka [marka] ,markieren', hárs [haurs] ,Haares' (Gen. Sing.). Möglicherweise kann $<1>$ noch stimmhaft vor $<\mathrm{p} k\rangle$ vorkommen: stelpa [stelp ${ }^{\mathrm{h}} \mathrm{a}$ ] ,Mädchen', hálka [haulk ${ }^{\mathrm{h}} \mathrm{a}$, Glatteis', aber in den meisten Fällen ist $<1>$ stimmlos und der nachfolgende Verschlusskonsonant unaspiriert.

Die stimmlosen Nasale entstanden als letzter Schritt vor den alten Verschlusslauten $\langle\mathrm{p} \mathrm{t} \mathrm{k}\rangle$ wie in der Tabelle $7 \mathrm{zu}$ ersehen ist.

Tabelle 7. Minimalpaare stimmhafter und stimmloser Nasale und Liquide.

\begin{tabular}{|c|c|}
\hline$\langle\mathrm{mp}\rangle=\left[\mathrm{mp}_{\mathrm{o}}\right]$ & $\langle\mathrm{mb}\rangle=[\mathrm{mp}]$ \\
\hline kempa [chempa], Held' & kemba [c ${ }^{\mathrm{h}}$ empa] ,kämmen' \\
\hline$\langle\mathrm{nt}\rangle=[\mathrm{nt} \mathrm{n}]$ & $\langle\mathrm{nd}\rangle=[\mathrm{nt}]$ \\
\hline henta [henta] ,passen' & henda [henta], werfen' \\
\hline$\langle\mathrm{nk}\rangle=[\mathfrak{g} \mathrm{k}]\left[\mathrm{n}_{0} \mathrm{c}\right]$ & $\langle\mathrm{ng}\rangle=[\mathrm{nk}][\mathrm{nc}]$ \\
\hline banka [paunka] ,klopfen' & banga [paunka] ,ängstlich' (Akk.) \\
\hline
\end{tabular}




\begin{tabular}{|c|c|}
\hline banki [paunci] ,Bank' & banginn [paunin], ängstlich' \\
\hline$\langle\mathrm{mt}\rangle=[\mathrm{mt}]$ & $\langle\mathrm{md}\rangle=[\mathrm{mt}]$ \\
\hline gleymt [kleimt] ,vergessen' (Part.N.) & gleymd [kleimt], vergessen' (Part.F.) \\
\hline$\langle\mathrm{lt}\rangle=[1 \mathrm{t} t]$ & $\langle\mathrm{Id}\rangle=[\mathrm{lt}]$ \\
\hline valta [valta] ,wälzen' & valda [valta] ,verursachen' \\
\hline$\langle\mathrm{rk}\rangle=[\mathrm{r} \mathrm{k}]$ & $\langle\mathrm{rg}\rangle=[\mathrm{rk}]$ \\
\hline marka [marka] ,markieren' & marga [marka], viele' (m.Akk.P1.) \\
\hline
\end{tabular}

Diese Änderung erfasste allerdings nicht das gesamte geographische Gebiet. In einem nördlichen Gebiet blieben die stimmhaften Nasale erhalten und der nachfolgende Verschlusskonsonant blieb aspiriert: lampi [lamp ${ }^{\mathrm{h}_{\mathrm{I}}}$, Lampe', henta [hent ${ }^{\mathrm{h}} \mathrm{a}$ ] ,passen, günstig sein', banka [paußk ${ }^{\text {ha }}$, klopfen' usw.

\section{Schlussfolgerung}

Die konservative Orthographie des Isländischen verdeckt die meisten Lautveränderungen, die in diesem Beitrag beschrieben werden. Es gibt auch keine Lautbeschreibungen, die uns eindeutig ermöglichen die Lautveränderungen zu datieren. Deshalb ist eine absolute Chronologie nicht möglich.

Die Änderungen im Vokalsystem sind nach allgemeiner Auffassung im 16. Jahrhundert abgeschlossen worden (Pórólfsson 1929), aber deren Anfang könnte vielleicht sogar im 14. Jahrhundert stattgefunden haben.

Es ist wahrscheinlich, dass die Änderungen im Konsonantensystem in der gleichen Zeit stattgefunden haben. Das kann daraus erschlossen werden, dass gewisse konsonantische Änderungen davon abhängig sind, ob der vorangehende Vokal lang oder kurz war. Aber wir können nur eine relative Chronologie erstellen. Wenn wir davon ausgehen, dass die ältesten Phänomene die größte Ausbreitung haben, wäre dịe relative Chronologie die folgende:

1. Erster Schritt ist der Verlust der Stimmhaftigkeit der alten $\langle\mathrm{b} d \mathrm{~g}\rangle$.

2. Zweiter Schritt ist die Entstehung des Hauchs [h] vor den ehemals langen $<p p$ tt $\mathrm{kk}>$ und den langen Gruppen $\langle\mathrm{pl}$ pn $\mathrm{tl}$ tn $\mathrm{kl} \mathrm{kn}\rangle$. Gleichzeitig entstehen die Verschlusskonsonanten in den Gruppen $\langle\mathrm{fl} \mathrm{fn} \mathrm{rl} \mathrm{rn}>$ und die langen $\langle 11 \mathrm{nn}\rangle$ werden nach ehemals langen Vokalen zu Konsonantengruppen [tl tn].

3. Dritter Schritt ist die Entstehung der stimmlosen Liquiden, die noch nicht das ganze Sprachgebiet erfasst hat. 
4. Vierter Schritt ist die Entstehung der stimmlosen Nasale, die in vorkonsonantischer Stellung nicht im ganzen Sprachgebiet vorkommen.

Für die beiden Schritte drei und vier kann gesagt werden, dass die Ausbreitung noch nicht ganz abgeschlossen ist, da das ganze Sprachgebiet noch nicht erfasst wurde. Es ist aber vorauszusehen, dass dies geschehen wird, da die überwiegende Mehrheit der Bevölkerung stimmlose Nasale und Liquide in vorkonsonantischer Stellung verwendet.

Isländisch hat eine große Lautverschiebung gehabt, die absolut vergleichbar mit Lautverschiebungen ist, die in anderen germanischen Sprachen stattgefunden haben (Goblirsch 2001). Das Ergebnis ist ein typologisch sehr spezifisches Konsonantensystem, das als Folge des Stimmhaftigkeitsverlustes der Verschlusskonsonanten entstanden ist. Es musste nicht zu diesem Ergebnis führen, wie wir daraus erkennen können, dass eine andere germanische Sprache, das Dänische, die stimmhaften Verschlusskonsonanten verloren hat, ohne dass stimmlose Nasale und Liquide entstanden. Wir können letztendlich nur den Weg einer Lautveränderung beschreiben, aber ihre Ursachen kennen wir nicht. Alle Lautveränderungen, die hier beschrieben werden, sind gut bekannt, aber bisher sind sie nicht im Zusammenhang gesehen worden. Wenn wir sie im Zusammenhang betrachten, ergeben sich neue Möglichkeiten der Erklärung, die noch nicht erschöpft worden sind.

\section{Bibliographie}

BERG, Thomas: An experimental study of syllabification in Icelandic. Nordic Journal of Linguistics 24, 71-106 (2001)

BESSASON, Haraldur: A few specimens of North American-Icelandic. Scandinavian Studies 39, 115-146 (1967)

GobLIRSCH, Kurt Gustav: The Icelandic consonant shift in its Germanic context. Arkiv för Nordisk Filologi 116, $117-133(2001)$

GRoENKE, Ulrich: Diachrone Perdurabilität, Sprachpflege und Sprachplanung: Der Fall Isländisch. In, Language Reform, History and Future 2 (István Fodor and Claude Hagège eds.), 137-155. Helmut Buske Verlag, Hamburg 1983

PóRÓLFsSON, Björn K.: Kvantitetsomvæltningen i islandsk. Arkiv för Nordisk Filologi 45, 35-81 (1929) 


\section{Povzetek}

\section{PREMIKI GLASOV V ISLANDŠČINI}

Tema tega prispevka je véliki premik glasov, ki ga je doživela islandščina. Opisujejo se posamezne stopnje premika. Začetni korak premika, ki je zaobsegel tako soglasniški kot samoglasniški sestav, je bil v izgubi segmentalne kvantitete. To je imelo za posledico fonologizacijo kvalitete samoglasnikov in nastanek novih samoglasnikov. Kvantiteta se je obdržala kot lastnost zloga pod popolnoma drugačnimi pogoji kakor dotlej.

V sestavu soglasnikov se je izgubila zvenečnost pri zapornikih in sprožila plaz sprememb, ki so povzročile, da so nastali številni novi fonemi. Zlasti je treba opozoriti na nezveneče jezičnike in nezveneče nosnike, ki so kot varianta sicer navzoči v mnogih jezikih, zelo redko pa so fonemi. Zato je islandski sestav soglasnikov s tipološkega vidika med skandinavskimi jeziki nekaj posebnega.

Časovno se spremembe kvantitete navadno umeščajo v 16. stoletje, vendar so se spremembe lahko začele že prej. Vsekakor ne obstajajo v zvezi s spremembami v sestavu soglasnikov nikakršni opisi ali namigi v pisavi. Da se pa predložiti relativna kronologija, in sicer na podlagi razširjenosti posameznih sprememb. 